\title{
Article \\ Transfer of a Rational Crystal Contact Engineering Strategy between Diverse Alcohol Dehydrogenases
}

\author{
Brigitte Walla $^{1}{ }^{\circledR}$, Daniel Bischoff ${ }^{1}\left(\right.$, , Robert Janowski ${ }^{2}{ }^{\circledR}$, Nikolas von den Eichen ${ }^{1}$, Dierk Niessing ${ }^{2,3}$ \\ and Dirk Weuster-Botz ${ }^{1, *(1)}$ \\ 1 Institute of Biochemical Engineering, Technical University of Munich, Boltzmannstraße 15, \\ 85748 Garching, Germany; brigitte.walla@tum.de (B.W.); daniel.bischoff@tum.de (D.B.); \\ nikolas.eichen@tum.de (N.v.d.E.) \\ 2 Institute of Structural Biology, Helmholtz Zentrum München, Ingolstädter Landstraße 1, \\ 85764 Neuherberg, Germany; robert.janowski@helmholtz-muenchen.de (R.J.); \\ niessing@helmholtz-muenchen.de (D.N.) \\ 3 Institute of Pharmaceutical Biotechnology, Ulm University, James Franck Ring N27, 89081 Ulm, Germany \\ * Correspondence: dirk.weuster-botz@tum.de
}

Citation: Walla, B.; Bischoff, D.; Janowski, R.; von den Eichen, N.; Niessing, D.; Weuster-Botz, D. Transfer of a Rational Crystal Contact Engineering Strategy between Diverse Alcohol Dehydrogenases. Crystals 2021, 11, 975. https:// doi.org/10.3390/cryst11080975

Academic Editor: Abel Moreno

Received: 22 July 2021

Accepted: 14 August 2021

Published: 17 August 2021

Publisher's Note: MDPI stays neutral with regard to jurisdictional claims in published maps and institutional affiliations.

Copyright: (C) 2021 by the authors. Licensee MDPI, Basel, Switzerland. This article is an open access article distributed under the terms and conditions of the Creative Commons Attribution (CC BY) license (https:// creativecommons.org/licenses/by/ $4.0 /)$.

\begin{abstract}
Protein crystallization can serve as a purification step in biotechnological processes but is often limited by the non-crystallizability of proteins. Enabling or improving crystallization is mostly achieved by high-throughput screening of crystallization conditions and, more recently, by rational crystal contact engineering. Two selected rational crystal contact mutations, Q126K and T102E, were transferred from the alcohol dehydrogenases of Lactobacillus brevis ( $L b \mathrm{ADH})$ to Lactobacillus kefir (LkADH). Proteins were expressed in E. coli and batch protein crystallization was performed in stirred crystallizers. Highly similar crystal packing of $L k A D H$ wild type compared to $L b A D H$, which is necessary for the transfer of crystal contact engineering strategies, was achieved by aligning purification tag and crystallization conditions, as shown by X-ray diffraction. After comparing the crystal sizes after crystallization of $L k A D H$ mutants with the wild type, the mean protein crystal size of $L k \mathrm{ADH}$ mutants was reduced by $40-70 \%$ in length with a concomitant increase in the total amount of crystals (higher number of nucleation events). Applying this measure to the $L k A D H$ variants studied results in an order of crystallizability T102E $>$ Q126K > LkADH wild type, which corresponds to the results with $\mathrm{LbADH}$ mutants and shows, for the first time, the successful transfer of crystal contact engineering strategies.
\end{abstract}

Keywords: technical protein crystallization from impure sources; rational crystal contact engineering; downstream processing; crystal image analysis

\section{Introduction}

Nowadays, protein crystallization is mainly used for crystallography to obtain the structural information of biological macromolecules. However, growing well-diffracting protein crystals for X-ray analysis is still challenging. There are numerous commercially available high-throughput screening kits that can be used to vary extrinsic conditions like $\mathrm{pH}$, buffer type, ionic strength, or precipitant type for receiving protein crystals [1]. However, the optimization of protein crystallization remains a mainly empirical process [2] and improved crystallization conditions apply almost exclusively to a specific protein or protein family. Thus, the protein itself might be the most important variable for a more general optimization of crystallization [3].

Changing intrinsic characteristics by protein engineering to obtain protein crystals (for Xray crystallography) has been conducted since the early 1990s [4-7]. Exchanging amino acids at the solvent-exposed surface of proteins has been investigated in several studies [3,8-12]. Derewenda et al. developed the surface entropy reduction (SER) strategy, in which exposed amino acids with high conformational entropy (Lys, Glu, Gln) are exchanged to an amino 
acid with low conformational entropy (Ala), in order to enhance crystallizability $[3,9,13]$. This approach was expanded by suggesting further amino acids for the exchange, including tyrosine and threonine [10]. An alternative approach to improve crystallization by engineering the solvent-exposed protein surface is to introduce metal binding sites between symmetry-related macromolecules by inserting aspartic acid or histidine-cysteine pairs for coordinating heteroatoms $\left(\mathrm{Ca}^{2+}, \mathrm{Zn}^{2+}\right)$ [14-16]. Furthermore, introduced cysteines at the protein surface can form disulfide bonds for dimer stabilization as a precursor for nucleation [17]. However, these studies were primarily aimed at producing large, singular crystals sufficient for structural analysis by X-ray crystallography.

A further interesting field of application for protein crystallization is its use as a purification and/or capture step in industrial downstream processing [18]. Protein purification by diffusion controlled preparative chromatography is a cost-intensive bottleneck in downstream processing. Consequently, the replacement of this purification step with a comparatively less cost-intensive crystallization step offers great economical potential (reviewed in $[18,19])$. In addition, protein crystallization allows a dense and highly concentrated, as well as pure formulation with a longer shelf life and sustained enzymatic activity [20-22]. Probably the most well-known technical application of protein crystallization is the production of crystalline recombinant insulin [21,23]. However, despite advantages for technical applications, protein crystallization has so far barely been integrated in biotechnological downstream processes [24].

Previous studies focused on enforcing intermolecular interactions during crystallization specifically at crystal contact areas to increase protein crystallization for technical purposes. Studies were performed with the technically relevant enzyme Lactobacillus brevis alcohol dehydrogenase $(\mathrm{LbADH})$ [24-27]. In addition to the general SER strategy of Derewenda et al., aromatic interactions as well as electrostatic interactions at specific preselected crystal contacts were investigated. This rational protein engineering at crystal contact areas resulted in $\mathrm{LbADH}$ mutants with enhanced crystallizability, compared to LbADH wild type, without changing enzymatic activity. In the same study, "enhanced crystallizability" was defined by an increased number of protein crystals in batch processes at fixed process times, concomitant to increased nucleation rate, reduced induction time of crystallization, and a reduced time span until crystallization equilibrium [24]. In order to be able to trace changes in crystallizability back to this distinct amino acid exchange and avoid unintended changes of crystal contact areas compositions, the formation of highly similar crystal contact areas is a prerequisite [25].

The aim of this study is to transfer protein engineering strategies at crystal contact areas, which were developed for $\mathrm{Lb} \mathrm{ADH}$, to a different protein, the Lactobacillus kefir alcohol dehydrogenase ( $L k \mathrm{ADH}$ ) with an amino acid sequence homology of $88.5 \%$ (Supplementary Figure S1). The purpose is to demonstrate that rational protein engineering at crystal contacts leading to enhanced crystallizability can be transferred to homologous proteins. However, similar to crystal contact engineering, the prerequisite of a rational transfer is high similarity in crystal packing. The initial focus of this study will be on the alignment of the crystal packing of $L k \mathrm{ADH}$ to $L b \mathrm{ADH}$. Based on the same crystal structure, the transfer of enhanced crystallizability will be demonstrated with the example of introduced electrostatic interactions at a selected crystal contact area. With regard to the application as a purification/capture step in downstream processing, stirred protein crystallization from clarified E. coli cell lysate will be investigated for the $L k A D H$ wild type, as well as LkADH mutants.

\section{Materials and Methods}

\subsection{Mutagenesis}

The enzyme Lactobacillus kefir alcohol dehydrogenase ( $L k \mathrm{ADH})$ was selected for the transfer of a successful crystal contact engineering strategy established with Lactobacillus brevis alcohol dehydrogenase ( $L b A D H)$. The His-tag and linker sequence of a $L k A D H$ variant (PDB ID: 4RF4, [28]) was replaced with a $\mathrm{N}$-terminal His 6 sequence, followed by a 
glycine-serine-glycine (GSG) linker sequence identical to the LbADH wild type studied before (PDB ID: 6H07, [27]). The E. coli codon optimized gene was synthesized by Integrated DNA Technologies Inc. (IDT, Leuven, Belgium) using Gibson assembly. The synthesized gene fragment was cloned into DNA expression plasmid pET28a(+) via restriction digest

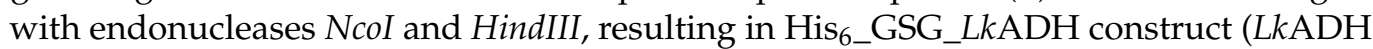
wild type). Site-directed mutagenesis was conducted for $L k A D H$ mutants T102E and Q126K according to the standard QuikChange PCR protocol. Primers were designed according to adaptions of Zheng et al. [29]. Partial overlapping primers used for LkADH mutant T102E were 5'-GATACCGAGACAGAAGAATGGC-3' and 5'-CAACTTCTATGGCTCTGTCTTC-3', for Q126K 5'-GGTATTAAGCGTATGAAAAA-CAAAGG-3' and 5'-CAGACCCATAATTCGCATAC-3' (mutagenized positions are denoted in boldface). After PCR product preparation (FastGene ${ }^{\circledR}$ Gel/PCR Extraction Kit, Nippon Genetics Europe GmbH, Düren, Germany) and DpnI digest, the amplified plasmid was transformed into $\mathrm{DH} 5 \alpha$ chemo-competent cells and inoculated on Luria-Bertani (LB) plate containing $35 \mu \mathrm{L} \mathrm{mL}^{-1}$ kanamycin. E. coli colonies, which incorporated the mutated plasmid, were selected by colony PCR (FastGene Optima HotStart Ready Mix, Nippon Genetics Europe GmbH, Düren, Germany). Plasmids of $L k A D H$ mutants were isolated via mini-prep and sequenced by IDT with standard primer petup and T7term.

\subsection{Protein production and Purification}

For heterogeneous protein production in E. coli, LkADH plasmid was transformed into expression strain BL21(DE3). Analogous to Nowotny et al. [25], $5 \mathrm{~mL}$ of preculture was grown for $18 \mathrm{~h}$ in terrific broth (TB) medium, containing $35 \mu \mathrm{L} \mathrm{mL}^{-1}$ kanamycin $(180 \mathrm{rpm}$, $\left.30{ }^{\circ} \mathrm{C}\right)$. Then, $0.5 \mathrm{~L}$ shake flasks filled with $100 \mathrm{~mL} \mathrm{~TB}$ medium $\left(35 \mu \mathrm{L} \mathrm{mL}^{-1}\right.$ kanamycin) were inoculated with preculture (final $\mathrm{OD}_{600}$ of 0.05 ) and incubated at $30{ }^{\circ} \mathrm{C}$ and $230 \mathrm{rpm}$ until an $\mathrm{OD}_{600}$ of $0.6-0.8$ was reached. Lkadh gene expression was induced by addition of $200 \mu \mathrm{M}$ isopropyl B-D-1-thiogalactopyranoside. After $20 \mathrm{~h}$ of $L k A D H$ protein production $\left(20^{\circ} \mathrm{C}, 230 \mathrm{rpm}\right)$, the cells were harvested by centrifugation $\left(1500 \times g, 4{ }^{\circ} \mathrm{C}, 10 \mathrm{~min}\right)$ and stored at $-20{ }^{\circ} \mathrm{C}$ for further use. For $\mu \mathrm{L}$-scale batch crystallization and enzymatic activity measurements (as described below), the harvested cells were disrupted via sonication, the cell lysate was centrifuged for clarification, the $L k A D H$ protein was purified by immobilized metal ion affinity chromatography (IMAC) and dialyzed against protein buffer (20 mM HEPES/ $\mathrm{NaOH}$ pH 7.0, $1 \mathrm{mM} \mathrm{MgCl} 2$ ).

\subsection{Protein Crystallization}

To obtain the $L k A D H$ wild type protein crystal structure, protein crystallization in $\mu \mathrm{L}-$ scale was conducted with purified protein according to Nowotny et al. [25] (0.1 M Tris/HCl pH 7.0, $75 \mathrm{mM} \mathrm{MgCl}_{2}$, polyethylene glycol (PEG) MME 550 or 3000). A theoretical molar extinction coefficient of 23,950 $\mathrm{M}^{-1} \mathrm{~cm}^{-1}$ (calculated with ProtParam; [30]) was used for protein concentration determination.

For parallel crystallization experiments with non-purified $L k A D H$ variants, harvested cells were processed according to Grob et al. [24]. Crystallization experiments with clarified (phosphate-buffered saline, pH 7.4) and subsequently dialyzed (20 mM HEPES/NaOH $\mathrm{pH} 7.0,1 \mathrm{mM} \mathrm{MgCl}_{2}$ ) cell lysate were conducted in up to eight parallel $5 \mathrm{~mL}$ stirred tank reactors with a stirrer speed of $150 \mathrm{rpm}$, as described in Smejkal et al. [31]. The parallel stirred tank crystallization reactors were adapted from Hebel et al. [32]. Pitched-blade impellers were printed using an MSLA 3D printer (Prusa SL1, Prusa Research a.s., Prague, Czech Republic) from biocompatible epoxy resin (Bio ENG3, 3Dreysns, Barcelona, Spain) with a layer height of $50 \mu \mathrm{m}$ and an initial exposure time of $35 \mathrm{~s}$ (exposure time for all subsequent layers was $12 \mathrm{~s}$ ). The stirrers were printed directly onto the build plate, with lateral supports for better build plate adhesion. After printing, the stirrers were cleaned and UV cured. Control of the stirrers was implemented with a custom-built control unit based on two six-axis stepper motor driver modules (TMCM6110, Trinamic, Hamburg, 
Germany) that controlled up to 12 stepper motors simultaneously. Microstepping enabled smooth stirrer rotation between 1 and $2000 \mathrm{rpm}$.

Before initiation of crystallization, the absorption of the samples was measured spectrophotometrically at $280 \mathrm{~nm}$ and adjusted to similar values with protein buffer. The samples were filtered through a $0.2 \mu \mathrm{m}$ polypropylene syringe filter to remove particles, which might function as heterogeneous crystallization nuclei. To adjust its temperature, $2.5 \mathrm{~mL}$ of filtered protein solution was transferred to a $5 \mathrm{~mL}$ crystallizer in a temperaturecontrolled water bath at $20^{\circ} \mathrm{C}$. Protein crystallization was initiated by the addition of $2.5 \mathrm{~mL}$ crystallization buffer $\left(0.1 \mathrm{M}\right.$ Tris $/ \mathrm{HCl} \mathrm{pH} \mathrm{7.0,50} \mathrm{mM} \mathrm{MgCl}_{2}, 200 \mathrm{~g} \mathrm{~L}^{-1}$ PEG MME 550 ) to the prepared protein solution, resulting in a protein concentration defined as initial protein concentration $\mathrm{c}_{0}$, and monitored for $48 \mathrm{~h}$ with regular intervals of sampling.

\subsection{Protein Analytics and Analysis of Crystal Photomicrographs}

The protein purity of collected IMAC fractions was validated by discontinuous sodium dodecyl sulphate polyacrylamide gel electrophoresis (SDS-PAGE). Enzymatic activity of purified $L k A D H$ variants was determined spectrophotometrically as described in Grob et al. [24]. The oxidation of acetophenone to R-1-phenyl alcohol catalyzed by $L k A D H$ was detected at $340 \mathrm{~nm}$ by stoichiometric reduction of $\mathrm{NADPH} / \mathrm{H}^{+}$to NADP ${ }^{+}$.

Stirred $L k A D H$ protein crystallization in $5 \mathrm{~mL}$ reactors was analyzed with bicinchoninic acid (BCA) protein assay for total protein concentration of the supernatant. A discontinuous SDS-PAGE was used for validation of similar $L k A D H$ protein concentrations between samples and densitometrical measurement of crystallization yield. To prevent further crystal growth or nucleation, protein solutions were diluted 10 -fold with protein buffer directly after sampling. For the analysis of crystal photomicrographs, samples were taken after $48 \mathrm{~h}$ of protein crystallization and diluted 10-fold. A $10 \mu \mathrm{L}$ drop placed in a crystallization plate (MRC UnderOil Crystallization Plate, SWISSCI, Neuheim, Switzerland) was monitored microscopically inside an incubator (KB115, Binder, Tuttlingen, Germany) at $20^{\circ} \mathrm{C}$. Photomicrographs were taken automatically by a digital camera (DS-Fi3) attached to a light microscope (Nikon Eclipse 50i with 4-fold objective (CFI Plan Fluor), Nikon, Düsseldorf, Germany), controlled by NIS Elements AR v.5.02 imaging software (Nikon, Düsseldorf, Germany).

\subsection{X-ray Diffraction and Data Refinement}

Diffraction data were collected at the Swiss Light Source (SLS) synchrotron beamline X06DA (PXIII), using a PILATUS 2M-F detector. All crystals were soaked in 25-30\% (v/v) ethylene glycol for cryo-protection and then mounted on a nylon fiber loop and flash-frozen in liquid nitrogen. X-ray data collection was performed at $100 \mathrm{~K}$ [33]. Diffraction spots were integrated and indexed with XDS [34]. Refinement was performed with the CCP4 suite [35]. The structure of the previously published LkADH wild type (PDB ID: 4RF4) was used as the search model for molecular replacement with PHASER [36] and the model was then refined using COOT [37] and REFMAC [38]. The final model and structure factors have been deposited in the Protein Data Bank (PDB) under identification code (ID) 7P36 for the LkADH wild type with transferred crystal packing and 7P7Y for the LkADH mutant Q126K. Quality indicators for the X-ray diffraction datasets and refinement results are given in Supplementary Table S1.

\section{Results and Discussion}

\subsection{Transfer of Crystal Packing from LbADH to LkADH}

Highly similar crystal packing of $L b A D H$ and $L k A D H$ is required to ensure that enhanced crystallizability of $L k A D H$ is caused by the transferred point mutation at the crystal contact. Since the LkADH variant described in the literature (PDB ID: 4RF4, [28]) does not form the same crystal contacts as $\mathrm{Lb} \mathrm{ADH}$, the sequence and crystallization conditions of $L k A D H$ were transferred from $L b A D H$. For this purpose, His-tag and linker of $L k A D H$ were designed analogously to the LbADH wild type used before (PDB ID: 6H07; [27]). 
Protein production and IMAC purification of $\mathrm{His}_{6}$ GSSG_LkADH (LkADH wild type) was implemented, resulting in purified and active $L k A D H$ wild type protein (SDS-PAGE and enzymatic activity are shown in Supplementary Figures S2 and S3). Crystallization of LkADH wild type in $\mu \mathrm{L}$-scale applying conditions similar to $\mathrm{LbADH}$ (PEG MME 550, magnesium chloride, Tris $/ \mathrm{HCl}, \mathrm{pH} 7.0$ ) yielded high quality protein crystals for X-ray diffraction (Supplementary Table S1). Analysis of X-ray diffraction data revealed a transformation of space group from C121 (PDB ID: 4RF4; crystallization conditions: PEG 3350, magnesium formate, $\mathrm{pH}$ 7.0) to I222. Thus, by aligning amino acid tags and crystallization conditions, the crystal structure of $L k \mathrm{ADH}$ could be transferred from a monoclinic crystal system (PDB ID: 4RF4) to an orthorhombic crystal system, as was observed with $\mathrm{LbADH}$ crystals. For the $\mathrm{LbADH}$, it has been proven previously that a transition from space group I222 to $P 2{ }_{1} 22{ }_{1}$ does not alter the crystal contact areas significantly [27]. An alignment of $\mathrm{LbADH}$ and $L k \mathrm{ADH}$ crystal structures of the tetramer reveals very high structural similarity (Figure 1) and highly similar crystal contacts (Supplementary Table S2), quantified by root-mean-square deviation (RMSD).

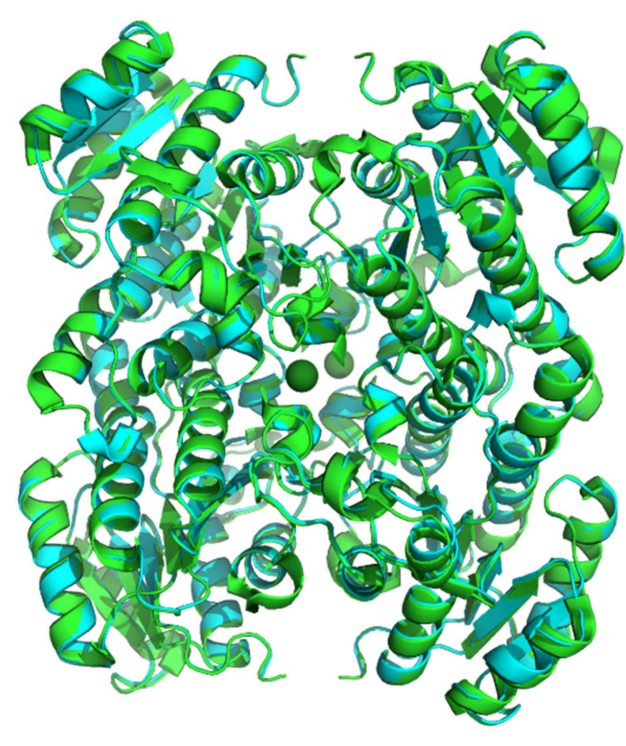

(a)

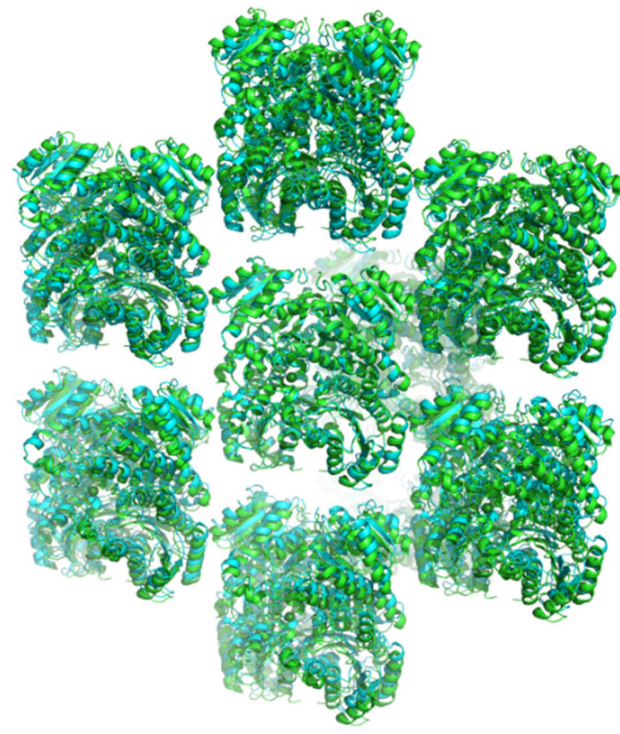

(b)

Figure 1. Structural alignment of $L k A D H$ (cyan; PDB ID: 7P36; $R_{\text {work }}=0.9 ; R_{\text {free }}=0.11$ ) and $L b A D H$ (green, PDB ID: 6H07) wild type (a) tetramers with $\mathrm{RMSD}_{\text {backbone }}=0.183 \AA$ and $\mathrm{RMSD}_{\text {side chain }}=0.212 \AA$ and $(\mathbf{b})$ with selected symmetry related molecules in the crystal structure. Figures were generated with PyMOL (v.2.3; Schroedinger). The homotetramers are complexing two $\mathrm{Mg}^{2+}$ ions (dark green).

This study aims to prove the transfer of rational protein engineering at crystal contacts to a homologous protein. The requirement of similar crystal contact areas for the transfer could be met by adjusting the purification tag and crystallization conditions for $L k A D H$ analogously to $\mathrm{Lb} \mathrm{ADH}$ resulting in the same crystal packing.

\subsection{Transfer of Mutations on Crystal Contacts from $\mathrm{LbADH}$ to $\mathrm{LkADH}$}

In order to study the transfer of rational crystal contact engineering strategies established for $\mathrm{LbADH}$, two out of four potential positions (K32, D54, T102, Q126) for the transfer of mutations on crystal contact areas of $\mathrm{LbADH}[24,25]$ were selected: T102 and Q126.

The choice of selecting T102 and Q126 was made by analyzing the crystal contacts of $L k \mathrm{ADH}$ and comparing them to $\mathrm{LbADH}$. In contrast to T102 and Q126, D54 is among the few amino acid positions that are noticeably different between $L b \mathrm{ADH}$ and $L k A D H$ (expressed by a relatively high contact RMSD value of D54, see Supplementary Table S2). The symmetric crystal contact of D54 is packed more densely in LkADH than in $L b A D H$, 
indicating different interactions at this contact for $L k A D H$. Position K32 in $L b A D H$ does not itself form a direct crystal contact, instead, mutant K32A contributes indirectly to the symmetric crystal contact at D54 by presumably reducing the screening of its electric field, as addressed in Nowotny et al. [25] and Hermann et al. [26]. Thus, the two crystal contact positions K32 and D54 were excluded as targets for the transfer of rational crystal contact engineering. The vicinity of the intermolecular crystal contact at position T102 and Q126 exhibits similar amino acid configurations for $\mathrm{LbADH}$ and $L k \mathrm{ADH}$ wild type (Figure 2; contact RMSD in Supplementary Table S2). More importantly, the interaction targets of the introduced mutations, which are K48 for T102E and E44 for Q126K, reveal similar orientations in $\mathrm{LbADH}$ and $\mathrm{LkADH}$ wild type crystal structure alignment. Additionally, it was shown for $\mathrm{LbADH}$ that the selected mutants T102E and Q126K generated a salt bridge in $\mathrm{Lb} \mathrm{ADH}$ crystals between negatively charged glutamic acid (E) and positive charged lysine (K) [26]. Thus, T102E and Q126K are the focus of this study.

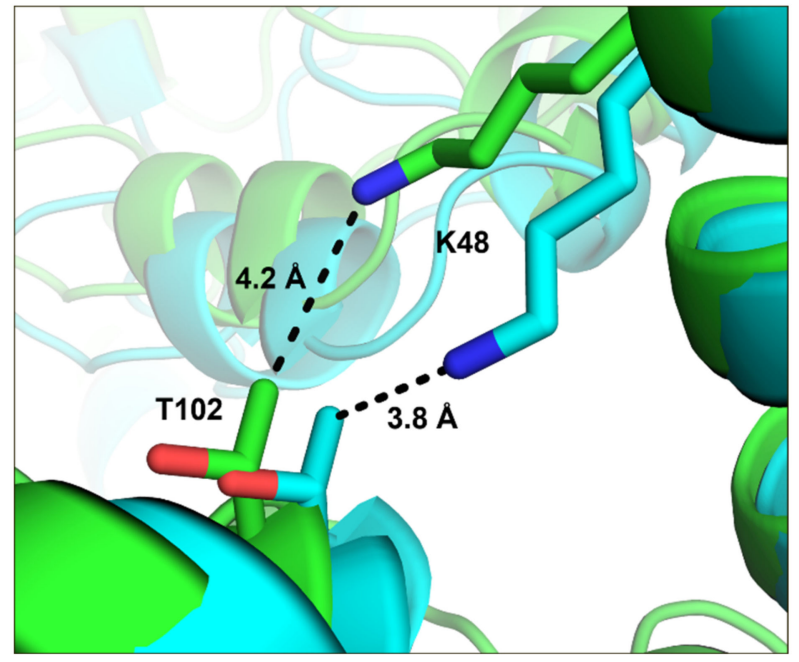

(a)

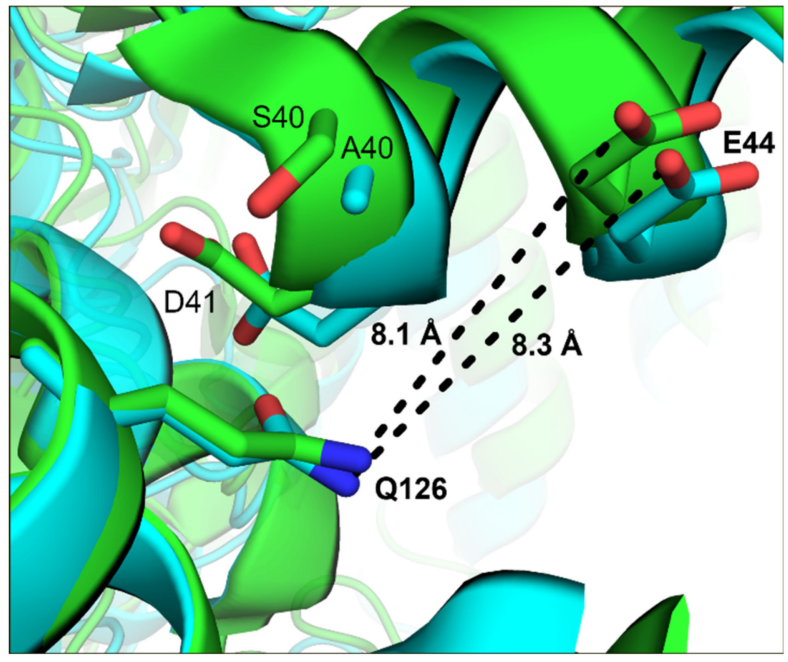

(b)

Figure 2. Illustration of $\mathrm{LkADH}$ (cyan) and $\mathrm{LbADH}$ (green) wild types' intermolecular crystal contacts at amino acid position (a) T102 and (b) Q126. Figures were generated and distances were calculated using PyMOL (v.2.3; Schroedinger).

Cloning of these two $L k A D H$ mutants was performed as described before. Protein production and downstream processing of mutant T102E and Q126K were accomplished, resulting in stable and pure protein solutions. $L k A D H$ protein solution purity after cell disruption, IMAC, dialysis and concentration as well as enzymatic activity of purified LkADH mutants is comparable to $L k A D H$ wild type (SDS-PAGE and enzymatic activity are shown in Supplementary Figures S2 and S3).

\subsection{Stirred Crystallization of Dialyzed and Clarified E. coli Cell Lysate}

Stirred batch crystallization studies with E. coli cell lysate were conducted with regard to the technical application of protein crystallization. First, stirred crystallization experiments were performed with clarified and additionally dialyzed E. coli cell lysate. During crystallization, the protein concentration of the supernatant decreases due to incorporation of $L k \mathrm{ADH}$ molecules into the protein crystals. The protein concentration in the supernatant of mutant T102E decreases more rapidly than with $L k A D H$ wild type within the first $5 \mathrm{~h}$ (Figure 3). Furthermore, the protein concentration of T102E equilibrates at a lower level after $21 \mathrm{~h}$ of crystallization compared to $L k \mathrm{ADH}$ wild type, a further characteristic for enhanced crystallizability and important for technical applications. 
(a)

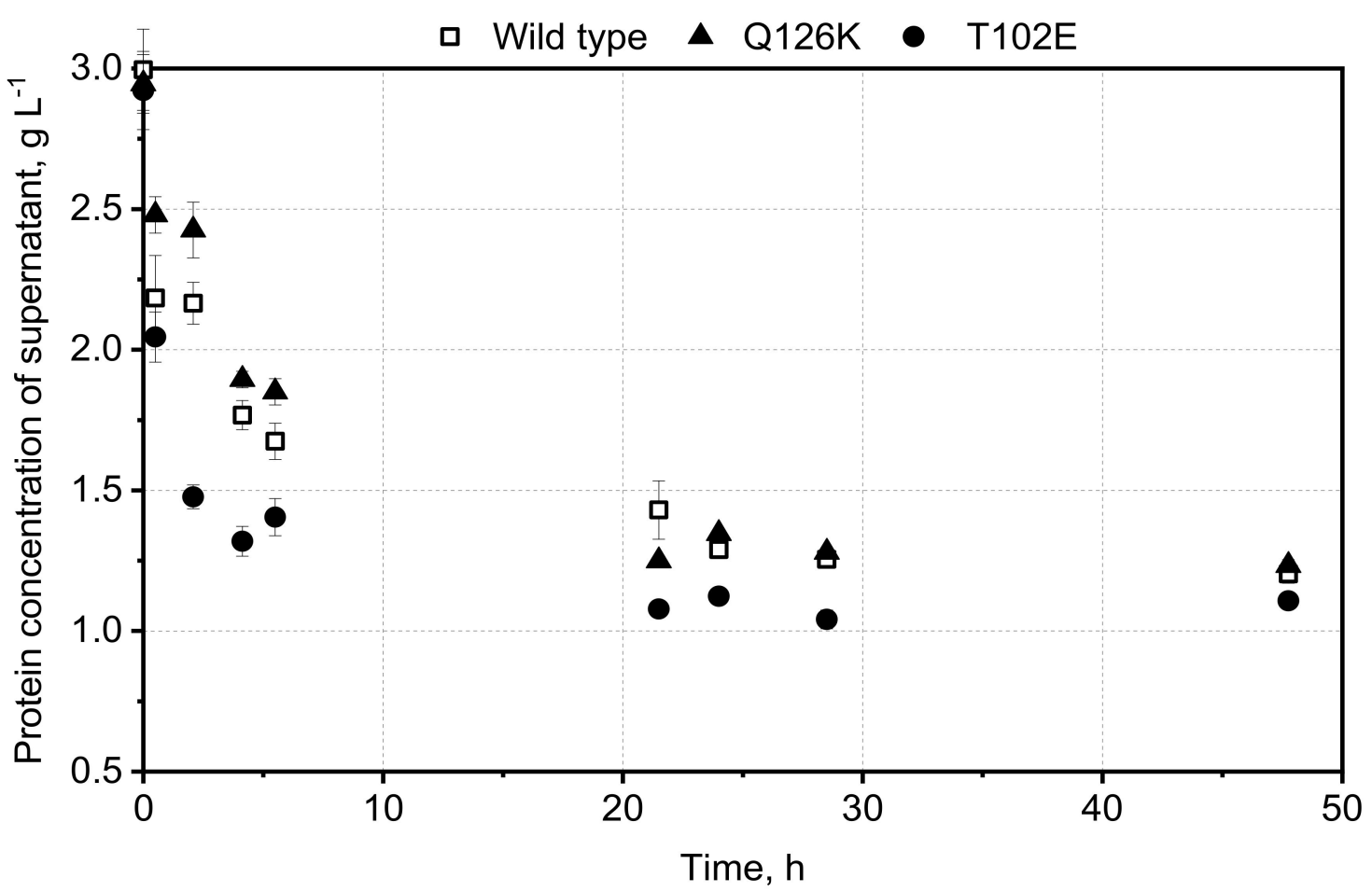

(b)
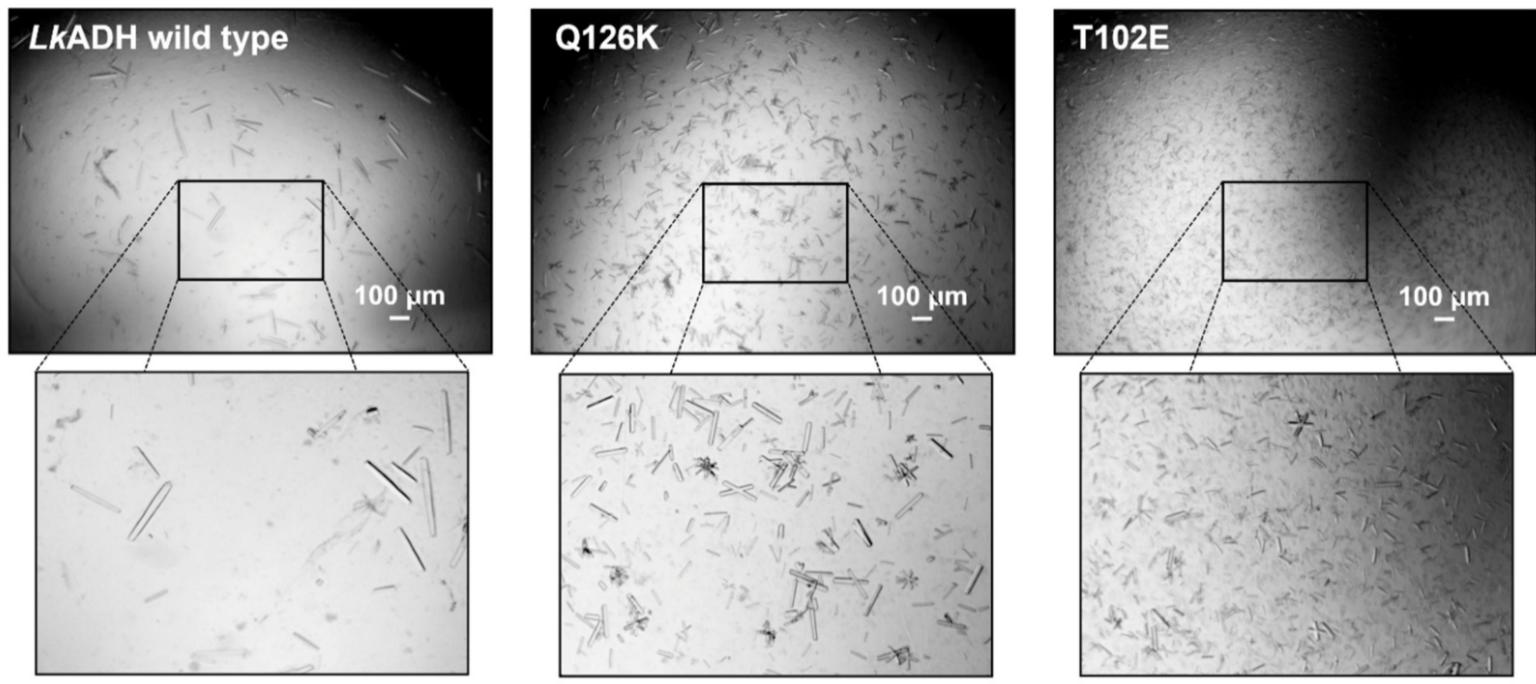

Figure 3. (a) Protein concentrations of clarified and dialyzed E. coli cell lysates of $L k A D H$ wild type and mutants T102E and Q126K. Batch crystallization was conducted in parallel stirred tank reactors $\left(\mathrm{V}=5 \mathrm{~mL}, \mathrm{n}_{\text {Stirrer }}=150 \mathrm{rpm}, 20^{\circ} \mathrm{C}, 100 \mathrm{~g} \mathrm{~L}-1\right.$ PEG MME 550). Protein concentration of supernatant was determined by BCA assay $(\mathrm{n}=3)$; $(\mathbf{b})$ Photomicrographs of LkADH wild type and mutants Q126K and T102E after $48 \mathrm{~h}$ of crystallization from clarified and dialyzed E. coli cell lysate in stirred tank reactors $\left(\mathrm{c}_{0}=3 \mathrm{~g} \mathrm{~L}^{-1}, \mathrm{~V}=5 \mathrm{~mL}, \mathrm{n}_{\text {Stirrer }}=150 \mathrm{rpm}, 20^{\circ} \mathrm{C}, 100 \mathrm{~g} \mathrm{~L}^{-1} \mathrm{PEG}\right.$ MME 550).

This reduced protein concentration at crystallization equilibrium and increased number of protein crystals of LkADH mutant T102E compared to the wild type LkADH has also been demonstrated previously for mutation T102E in $\mathrm{LbADH}$ (P2 ${ }_{1} 22_{1}$, PDB ID: 6Y0S) [24]. This indicates a successful transfer of a rational protein engineering strategy between homologous enzymes.

E. coli cell lysate still contains high amounts of host cell protein (HCP). Thus, crystallization yields quantified by total protein concentration measurements via BCA assay 
are underestimated. This is due to remaining amounts of HCP in the solution and results in apparent crystallization yields of only 59-63\% (Figure 4). Therefore, SDS-PAGE of the supernatants were performed before initiation of crystallization and at the end of the batch crystallization processes $(48 \mathrm{~h}$ ) and the results were compared densitometrically (Figure 4 ). The $L k A D H$ to HCP ratio in clarified and dialyzed cell lysate of $L k A D H$ mutants is similar to $L k A D H$ wild type. Comparing the SDS-PAGE bands of the $L k A D H$ variants before and after batch crystallization revealed a reduction in intensity of $L k A D H$ wild type protein concentration by $80 \%$; whereas the intensity of $L k A D H$ mutants Q126K and T102E decreased by $85 \%$, and about $95 \%$, respectively (evaluated with GelAnalyzer, v.19.1). The crystallization yield of about $95 \%$ estimated by SDS-PAGE showed improved crystallization of mutant T102E, whereas no significant yield differences were identified between mutant Q126K and wild type $L k A D H$.

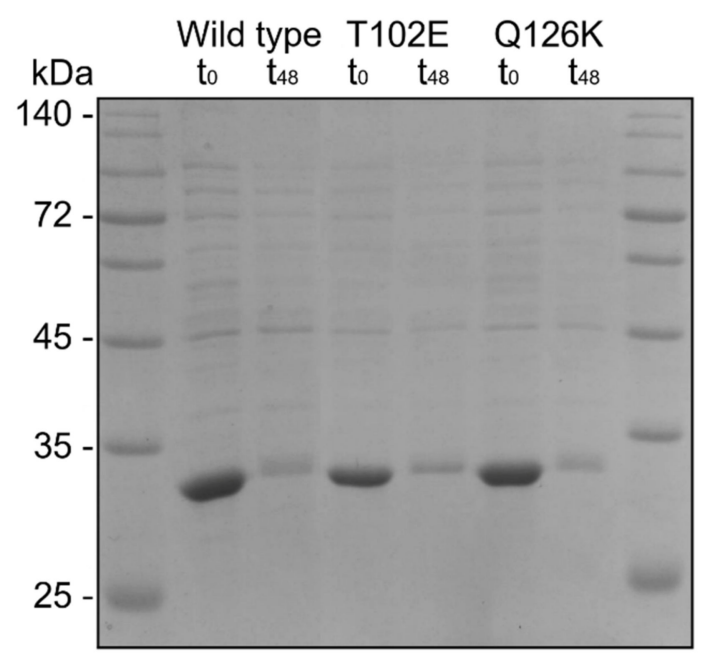

Figure 4. SDS-PAGE visualizing the protein content of clarified and dialyzed E. coli cell lysates of LkADH wild type and mutants T102E and Q126K. Depicted are samples of clarified and dialyzed E. coli cell lysates directly before crystallization initiation $\left(\mathrm{t}_{0}=0 \mathrm{~h}\right)$ and after $48 \mathrm{~h}\left(\mathrm{t}_{48}\right)$ of crystallization in stirred tank reactors $\left(\mathrm{V}=5 \mathrm{~mL}\right.$, $\mathrm{n}_{\text {Stirrer }}=150 \mathrm{rpm}, 20^{\circ} \mathrm{C}, 100 \mathrm{~g} \mathrm{~L}^{-1}$ PEG MME 550). LkADH variants are detected between $25-35 \mathrm{kDa}$, corresponding to its molecular monomer weight of $27.86 \mathrm{kDa}$ (calculated with ExPASy ProtParam).

\subsection{Evaluation of Crystal Size Differences between LkADH Variants}

A higher number of crystals and a decrease in crystal size were observed with the protein mutants, compared to the wild type $L k A D H$ in the photomicrographs of the batch crystallization processes with clarified and dialyzed E. coli cell lysates (Figure 3b). For the quantification of crystal size differences in length and width between the $L k A D H$ variants, the photomicrographs were analyzed manually with imaging software (NIS Elements AR v.5.02, Nikon, Düsseldorf, Germany). The resulting protein crystal size distributions are shown in Figure 5. The frequency density of $L k A D H$ wild type crystals follows approximately a log-normal distribution with its mean length at $97.1 \pm 29.5 \mu \mathrm{m}$ (mean width: $11.4 \pm 4.5 \mu \mathrm{m}$ ). A similar distribution is observed for the LkADH mutants, but with a shift of the mean crystal size to smaller crystal sizes by $40 \%$ for Q126K (mean length/width: $58.9 \pm 9.8 \mu \mathrm{m} / 10.2 \pm 2.9 \mu \mathrm{m}$ ) and by more than $70 \%$ for T102E (mean length/width: $27.5 \pm 6.4 \mu \mathrm{m} / 5.9 \pm 1.6 \mu \mathrm{m})$.

Comparing the crystals size to the wild type after stirred crystallization of $L k A D H$ mutants, the mean protein crystal size of $L k A D H$ mutants T102E and Q126K was reduced by more than $70 \%$ in length and $45 \%$ in width; and $40 \%$ in length and $10 \%$ in width, respectively (Figure 5), with a concomitant increase in the total amount of crystals (Figure 3b). This increase in the total number of crystals indicates a higher number of nucleation events for the mutants than occurred with the LkADH wild type. Stronger intermolecular interac- 
tions compared to the wild type (see electron density map of the crystal contact of LkADH wild type and mutant Q126K with an additional salt-bridge in Supplementary Figure S4) can therefore be assumed to lead to an increased number of nucleation events. This correlation was already observed for $\mathrm{Lb} \mathrm{ADH}$ mutants T102E and Q126K and supported on a molecular level by calculations of molecular dynamics free energy simulations $[24,26]$.
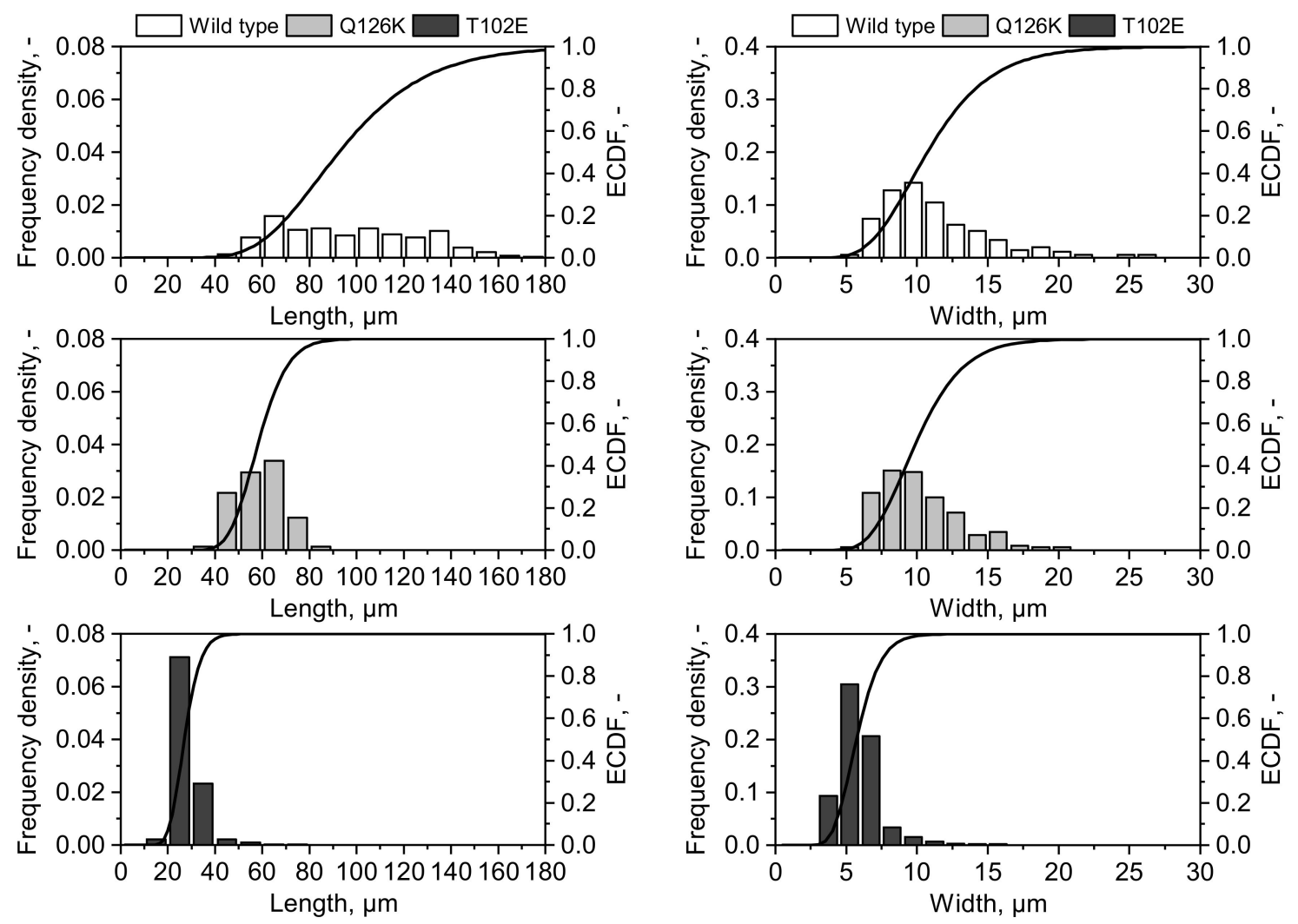

Figure 5. Protein crystal size distribution of $L k A D H$ wild type and mutants T102E and Q126K (Bar: Frequency density distribution; Line: Empirical cumulative distribution function). The distributions of length (left) and width (right) of the protein crystals formed after $48 \mathrm{~h}$ batch crystallization from clarified and dialyzed E. coli cell lysate in stirred tank reactors $\left(c_{0}=3 \mathrm{~g} \mathrm{~L}^{-1}, \mathrm{~V}=5 \mathrm{~mL}, \mathrm{n}_{\text {Stirrer }}=150 \mathrm{rpm}, 20^{\circ} \mathrm{C}, 100 \mathrm{~g} \mathrm{~L}^{-1} \mathrm{PEG} \mathrm{MME} 550\right)$. The frequency density distribution of protein crystals in specific length/width classes in $\mu \mathrm{m}$ was evaluated with OriginPro $2020(n=335)$.

\subsection{Stirred Crystallization of Clarified E. coli Cell Lysate}

All batch protein crystallization processes were reproduced in the stirred tank reactors, without dialysis, after cell disruption and clarification (Figure 6). Due to the increased $\mathrm{HCP}$ concentrations without dialysis, no crystallization of $L k \mathrm{ADH}$ wild type was observed within $48 \mathrm{~h}$, but the mutants Q126K and T102E formed crystals as before.

Crystallization kinetics of the batch processes with clarified and dialyzed E. coli cell lysates, as shown before (Figure 3), as well as final crystallization yields estimated by SDSPAGE (Figure 4), showed no significant differences in crystallizability between LkADH wild type and the mutant Q126K. Clear differences were identified in the final protein crystal size distributions of both proteins, showing an increase in nucleation events with LkADH mutants compared to LkADH wild type under the conditions studied (Figure 5). A small variation of these conditions caused by batch crystallizations of clarified E. coli lysates without dialysis resulted in no nucleation events within $48 \mathrm{~h}$ with the LkADH wild type (Figure 6). As the crystallizability of proteins is not well-defined, it may be concluded that comparing final protein crystal size distribution and the number of crystals may be a practical measure for defining an order of crystallizability: An increased final crystal number with reduced crystal sizes means better crystallizability compared to other 
proteins under the same crystallization conditions. Applying this measure to the LkADH proteins studied results in an order of crystallizability: T102E > Q126K > LkADH wild type. A comparison of this ranking with $\mathrm{LbADH}[24,26]$ reveals the identical order of crystallizability, further validating the rational approach of the protein engineering strategy transfer between homologous enzymes.
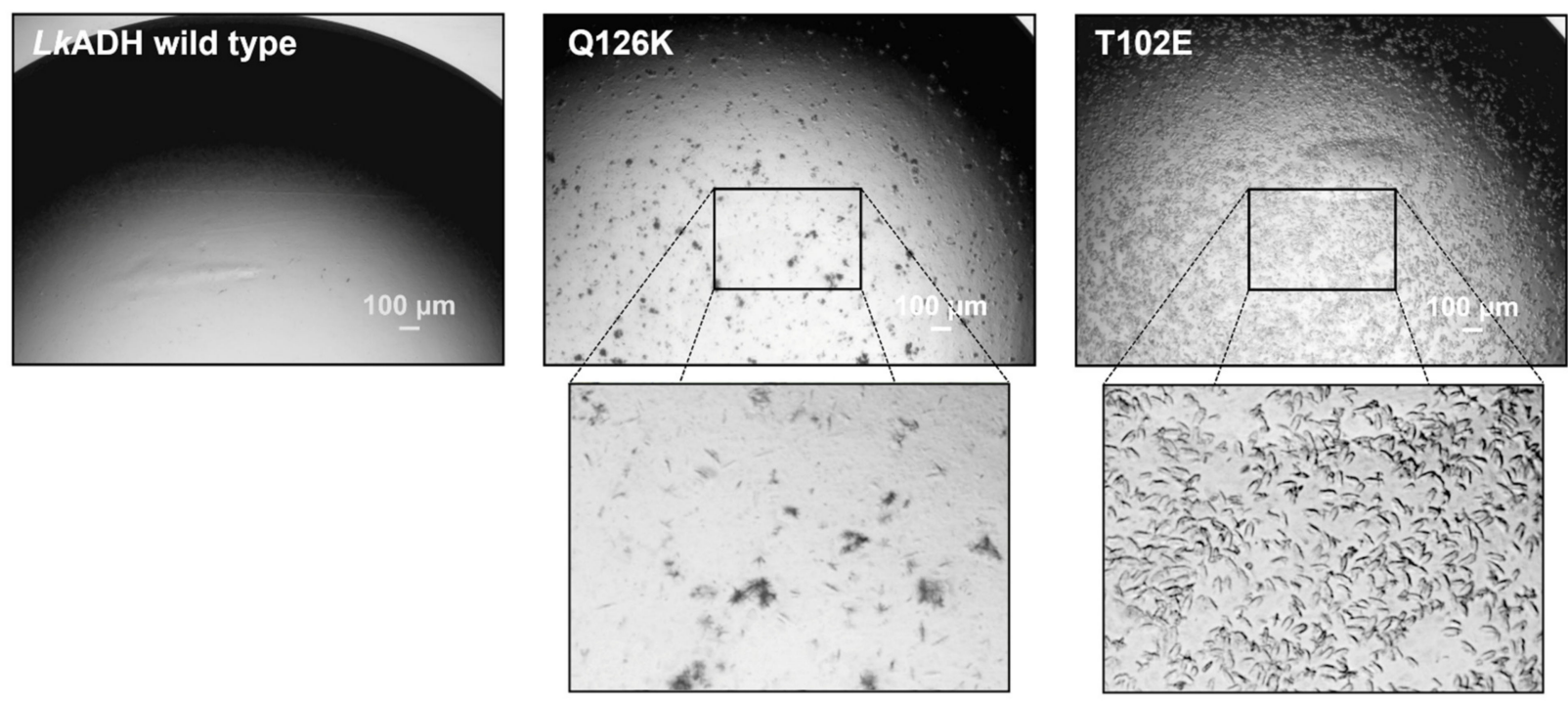

Figure 6. Photomicrographs of $L k A D H$ wild type and mutants Q126K and T102E after $48 \mathrm{~h}$ of crystallization from clarified E. coli

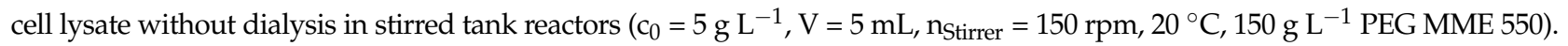

\subsection{Comparison of LkADH and LbADH Crystal Contacts at Position Q126 on a Molecular Level}

For further evidence of effective transfer of crystal contact mutations, the introduced single amino acid mutations in $L k A D H$ will be considered at molecular level and compared to the respective $\mathrm{LbADH}$ mutants. Since the protein crystallization of $L k A D H$ mutant T102E occurs so rapidly, the crystals obtained were too small for X-ray analysis using non-microfocus X-ray beamline. Therefore, the X-ray crystal structure of LkADH mutant Q126K is discussed below.

Comparing the position 126 in LkADH wild type (Figure 2) and mutant Q126K (Figure 7a), the interacting amino acid has changed. In contrast to Q126 interacting weakly with D41, Q126K developed a strong interaction with E44, resulting in a salt bridge between the ammonium group of the lysine side chain $(\mathrm{K})$ and the carboxylate group of glutamic acid (E). This establishment of interaction with E44 is also observed for LbADH mutant Q126K (Figure 7b).

Comparison of the crystal contact at position Q126K reveals the same interaction partner for both $L b \mathrm{ADH}$ and $L k \mathrm{ADH}$. The interacting amino acids K126 and E44 in $L k A D H$ are more closely located (2.8 $\AA$ distance), indicating a stronger interaction than in $L b A D H$ Q126K (3.9 A distance, evaluated with PyMOL, v.2.3.; Schroedinger). Additionally, the LbADH Q126K crystal structure presents two possible conformations for both amino acids E44 and K126, suggesting a more flexible interaction at this position. One of the possible conformations of E44 is even facing away from K126. Recent molecular dynamic studies determined the formation of the salt bridge between $\mathrm{K} 126$ and $\mathrm{E} 44$ in about $65 \%$ of the simulated time frames for $\mathrm{LbADH}$ [26]. Contrary to $\mathrm{LbADH}$, the crystal structure of LkADH mutant Q126K reveals one conformation for the K126-E44 crystal contact with E44 pointing directly to K126, which is well-defined in the electron density map (Supplementary Figure S4b). This single conformation indicates a stronger intermolecular interaction, suggesting an enhanced crystallizability for the transfer of mutant Q126K from $L b A D H$ to $L k \mathrm{ADH}$. To confirm this assumption, further calculations of molecular dynamics free energy simulations of this crystal contact are necessary. To conclude, the transfer of interacting 
amino acids in mutant Q126K was successfully transferred from $L b A D H$ to $L k A D H$, with a tendency towards a stronger crystal contact formation in $L k \mathrm{ADH}$.

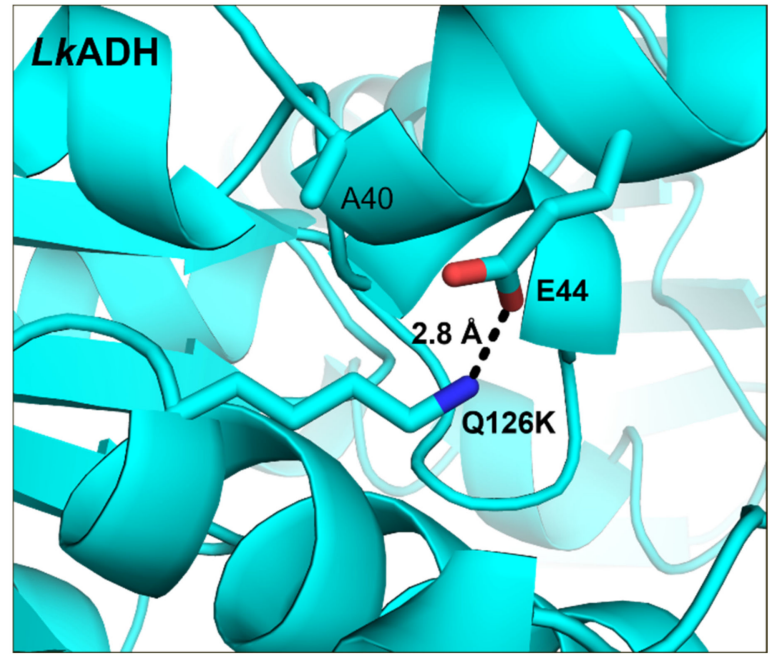

(a)

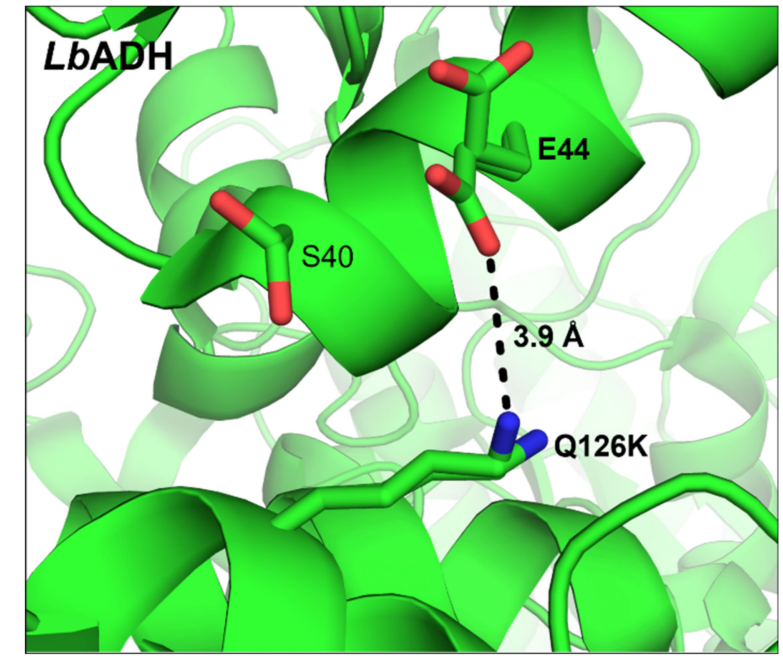

(b)

Figure 7. Illustration of crystal contact Q126K in (a) LkADH (PDB ID: 7P7Y) and (b) LbADH (PDB ID: 6Y0Z) with interaction partner E44. Distances were calculated using PyMOL (v.2.3; Schroedinger).

Based on the evaluation of stirred crystallization experiments and considerations at the molecular level, $\mathrm{LkADH}$ mutants T102E and Q126K are more favorable for technical applications than $L k A D H$ wild type in terms of crystallizability. This is because the main objective for technical protein crystallization is to achieve rapid and efficient protein crystallization [39] to increase the space-time yield of the process. Considering the technical application of protein crystallization from impure sources as a purification step, future work should investigate the purity of the crystals.

This study shows that the results of rational crystal contact engineering of $L b A D H$ can be transferred successfully to the homologous $L k A D H$. To advance the establishment of a more general approach to enhance protein crystallization by rational crystal contact engineering, developed strategies for $\mathrm{Lb} \mathrm{ADH}$ and $\mathrm{LkADH}$ will be extended further to non-homologous proteins.

Supplementary Materials: The following are available online at https: / www.mdpi.com/article / 10.3390 / cryst11080975/s1, Figure S1: Sequence alignment of $L k A D H$ and $L b A D H$ wild type with an amino acid sequence homology of $88.5 \%$. The optimal global alignment was generated with the Needleman-Wunsch algorithm (EMBOSS, output format: pair, matrix: BLOSUM62) [1]. Identical amino acids are marked with an asterisk. Crystal contacts (distance between amino acids $<4 \AA$ ) are marked for $L k A D H$ (cyan) and $L b A D H$ (green). The positions selected for mutations, T102 and Q126, are underlined. RMSD of the crystal contacts are listed in Supplementary Table S2. Figure S2: Illustration of protein purity analysis using SDS-PAGE of LkADH wild type (WT) and mutants Q126K (K) and T102E (E). The proteins purified by IMAC were loaded onto a $12.5 \%$ SDS gel (2.5 $\mu \mathrm{g}$ protein) under reducing conditions. Bands between 25-35 kDa correspond to LkADH monomers. Figure S3: Maximum relative enzymatic activity of $L k A D H$ mutants Q126K and T102E compared to $L k A D H$ wild type. Purified protein solutions of $L k A D H$ variants were adjusted to $6 \mathrm{mg} \mathrm{L}^{-1}$. Enzymatic activity was measured for $10 \mathrm{~min}$ at $340 \mathrm{~nm}\left(45^{\circ} \mathrm{C}\right)$ with addition of $180 \mu \mathrm{L}$ buffer $(20 \mathrm{mM}$ HEPES, $\mathrm{pH} 7.0 ; 1 \mathrm{mM} \mathrm{MgCl}_{2}$ ) containing $0.5 \mathrm{mM} \mathrm{NADPH}$ and $10 \mathrm{mM}$ acetophenone to a final volume of $200 \mu \mathrm{L}(\mathrm{n}=3)$. Figure S4: Electron density map (blue) of $L k A D H$ crystal contact at position (a) Q126 and (b) Q126K with well-defined electron density of the lysine and glutamic acid side chain. The map represents the structure factor amplitude difference $2 \mathrm{~F}_{\mathrm{O}}-\mathrm{F}_{\mathrm{C}}$ with a contour level of $1.0 \mathrm{\sigma}$ (calculated with REFMAC [2]). Table S1: Data collection and refinement statistics of $\mathrm{X}$-ray diffraction experiments of $L k \mathrm{ADH}$ wild type and mutant Q126K crystals (values in parentheses are for highest resolution 
shell). Table S2: RMSD side chain of crystal contacts present in both $L k A D H$ and $L b A D H$ wild type and calculated after side chain and backbone alignment of one tetramer. Crystal contacts were defined by a distance $<4 \AA$ A between amino acid residues of neighboring tetramers. The contact $\mathrm{RMSD}_{\text {side chain }}$ was then calculated by alignment of those amino acid residues forming a contact with PyMOL (v.2.3.; Schroedinger). Therefore, a smaller contact RMSD value indicates, that the corresponding crystal contacts in $L b \mathrm{ADH}$ and $L k \mathrm{ADH}$ wild type crystals are locally more similar.

Author Contributions: Conceptualization, B.W. and D.B.; methodology, B.W. and N.v.d.E.; validation, B.W. and D.B.; investigation, B.W.; X-ray resources and diffraction experiments, R.J. and D.N.; writing - original draft preparation, B.W.; writing — review and editing, B.W., D.B., D.W.-B., and R.J.; supervision, D.W.-B. All authors have read and agreed to the published version of the manuscript.

Funding: This research was funded by the German Research Foundation (DFG): research project WE2715/14-2 within the framework of priority program SPP 1934.

Data Availability Statement: All X-ray crystal structure data in this study have been deposited and are available in the Protein Data Bank under the identification codes 7P36 and 7P7Y. All other data generated or analyzed during this study are included in this article and the Supplementary Material or are available from the corresponding author upon reasonable request.

Acknowledgments: The authors thank the X-ray Crystallography Platform at the Helmholtz Zentrum München. Support of B.W., D.B. and N.v.d.E. from the TUM Graduate School is gratefully acknowledged.

Conflicts of Interest: The authors declare no conflict of interest.

\section{References}

1. Newman, J.; Fazio, V.J.; Lawson, B.; Peat, T.S. The C6 Web Tool: A Resource for the Rational Selection of Crystallization Conditions. Cryst. Growth Des. 2010, 10, 2785-2792. [CrossRef]

2. Weber, P.; Pissis, C.; Navaza, R.; Mechaly, A.E.; Saul, F.; Alzari, P.M.; Haouz, A. High-Throughput Crystallization Pipeline at the Crystallography Core Facility of the Institut Pasteur. Molecules 2019, 24, 4451. [CrossRef]

3. Longenecker, K.L.; Garrard, S.M.; Sheffield, P.J.; Derewenda, Z.S. Protein crystallization by rational mutagenesis of surface residues: Lys to Ala mutations promote crystallization of RhoGDI. Acta Crystallogr. Sect. D Biol. Crystallogr. 2001, 57, 679-688. [CrossRef]

4. Lawson, D.M.; Artymiuk, P.J.; Yewdall, S.J.; Smith, J.M.; Livingstone, J.C.; Treffry, A.; Luzzago, A.; Levi, S.; Arosio, P.; Cesareni, G.; et al Solving the structure of human $\mathrm{H}$ ferritin by genetically engineering intermolecular crystal contacts. Nature 1991, 349, 541-544. [CrossRef]

5. Braig, K.; Otwinowski, Z.; Hegde, R.; Boisvert, D.C.; Joachimiak, A.; Horwich, A.L.; Sigler, P.B. The crystal structure of the bacterial chaperonln GroEL at $2.8 \AA$. Nature 1994, 371, 578-586. [CrossRef]

6. Horwich, A. Working with Paul Sigler. Nat. Struct. Biol. 2000, 7, 269-270. [CrossRef] [PubMed]

7. McElroy, H.E.; Sisson, G.W.; Schoettlin, W.E.; Aust, R.M.; Villafranca, J.E. Studies on engineering crystallizability by mutation of surface residues of human thymidylate synthase. J. Cryst. Growth 1992, 122, 265-272. [CrossRef]

8. Derewenda, Z.S. The use of recombinant methods and molecular engineering in protein crystallization. Methods 2004, 34, 354-363. [CrossRef] [PubMed]

9. Derewenda, Z.S.; Vekilov, P.G. Entropy and surface engineering in protein crystallization. Acta Crystallogr. Sect. D Biol. Crystallogr. 2006, 62, 116-124. [CrossRef] [PubMed]

10. Cooper, D.R.; Boczek, T.; Grelewska, K.; Pinkowska, M.; Sikorska, M.; Zawadzki, M.; Derewenda, Z. Protein crystallization by surface entropy reduction: Optimization of the SER strategy. Acta Crystallogr. Sect. D Biol. Crystallogr. 2007, 63, 636-645. [CrossRef]

11. Derewenda, Z.S.; Godzik, A. The "Sticky Patch" Model of Crystallization and Modification of Proteins for Enhanced Crystallizability. Methods Mol. Biol. 2017, 1607, 77-115. [CrossRef]

12. Derewenda, Z.S. Protein crystallization in drug design: Towards a rational approach. Expert Opin. Drug Discov. 2007, 2, 1329-1340. [CrossRef] [PubMed]

13. Derewenda, Z.S. Rational protein crystallization by mutational surface engineering. Structure 2004, 12, 529-535. [CrossRef]

14. Laganowsky, A.; Zhao, M.; Soriaga, A.B.; Sawaya, M.R.; Cascio, D.; Yeates, T.O. An approach to crystallizing proteins by metal-mediated synthetic symmetrization. Protein Sci 2011, 20, 1876-1890. [CrossRef]

15. Devedjiev, Y.; Surendranath, Y.; Derewenda, U.; Gabrys, A.; Cooper, D.R.; Zhang, R.-g.; Lezondra, L.; Joachimiak, A.; Derewenda, Z.S. The Structure and Ligand Binding Properties of the B.subtilis YkoF Gene Product, a Member of a Novel Family of Thiamin/HMP-binding Proteins. J. Mol. Biol. 2004, 343, 395-406. [CrossRef]

16. Roosen-Runge, F.; Zhang, F.; Schreiber, F.; Roth, R. Ion-activated attractive patches as a mechanism for controlled protein interactions. Sci. Rep. 2014, 4, 7016. [CrossRef] 
17. Banatao, D.R.; Cascio, D.; Crowley, C.S.; Fleissner, M.R.; Tienson, H.L.; Yeates, T.O. An approach to crystallizing proteins by synthetic symmetrization. Proc. Natl. Acad. Sci. USA 2006, 103, 16230-16235. [CrossRef]

18. Hekmat, D. Large-scale crystallization of proteins for purification and formulation. Bioprocess Biosyst. Eng. 2015, 38, 1209-1231. [CrossRef] [PubMed]

19. Dos Santos, R.; Carvalho, A.L.; Roque, A.C.A. Renaissance of protein crystallization and precipitation in biopharmaceuticals purification. Biotechnol. Adv. 2017, 35, 41-50. [CrossRef]

20. Shenoy, B.; Wang, Y.; Shan, W.; Margolin, A.L. Stability of crystalline proteins. Biotechnol. Bioeng. 2001, 73, 358-369. [CrossRef] [PubMed]

21. Brange, J.; Vølund, A. Insulin analogs with improved pharmacokinetic profiles. Adv. Drug Deliv. Rev. 1999, 35, 307-335. [CrossRef]

22. Liu, Y.; Hou, H.; Li, J.; Cheng, Q.-D.; Zhang, X.; Zeng, X.-B.; Fiaz, A.; Wang, B.; Zhang, C.-Y.; Lu, Q.-Q.; et al. Direct Crystallization of Proteins from Impure Sources. Cryst. Growth Des. 2020, 20, 1694-1705. [CrossRef]

23. Harrison, R.G.; Todd, P.W.; Rudge, S.R.; Petrides, D.P. Bioseparations Science and Engineering, 2nd ed.; Oxford University Press: New York, NY, USA, 2003.

24. Grob, P.; Huber, M.; Walla, B.; Hermann, J.; Janowski, R.; Niessing, D.; Hekmat, D.; Weuster-Botz, D. Crystal Contact Engineering Enables Efficient Capture and Purification of an Oxidoreductase by Technical Crystallization. Biotechnol. J. 2020, e2000010. [CrossRef] [PubMed]

25. Nowotny, P.; Hermann, J.; Li, J.; Krautenbacher, A.; Klöpfer, K.; Hekmat, D.; Weuster-Botz, D. Rational Crystal Contact Engineering of Lactobacillus brevis Alcohol Dehydrogenase To Promote Technical Protein Crystallization. Cryst. Growth Des. 2019, 19, 2380-2387. [CrossRef]

26. Hermann, J.; Bischoff, D.; Grob, P.; Janowski, R.; Hekmat, D.; Niessing, D.; Zacharias, M.; Weuster-Botz, D. Controlling Protein Crystallization by Free Energy Guided Design of Interactions at Crystal Contacts. Crystals 2021, 11, 588. [CrossRef]

27. Hermann, J.; Nowotny, P.; Schrader, T.E.; Biggel, P.; Hekmat, D.; Weuster-Botz, D. Neutron and X-ray crystal structures of Lactobacillus brevis alcohol dehydrogenase reveal new insights into hydrogen-bonding pathways. Acta Crystallogr. Sect. F 2018, 74, 754-764. [CrossRef]

28. Noey, E.L.; Tibrewal, N.; Jiménez-Osés, G.; Osuna, S.; Park, J.; Bond, C.M.; Cascio, D.; Liang, J.; Zhang, X.; Huisman, G.W.; et al. Origins of stereoselectivity in evolved ketoreductases. Proc. Natl. Acad. Sci. USA 2015, 112, E7065-E7072. [CrossRef]

29. Zheng, L.; Baumann, U.; Reymond, J.L. An efficient one-step site-directed and site-saturation mutagenesis protocol. Nucleic Acids Res. 2004, 32, e115. [CrossRef]

30. Gasteiger, E.H.C.; Gattiker, A.; Duvaud, S.; Wilkins, M.R.; Appel, R.D.; Bairoch, A. Protein Identification and Analysis Tools on the ExPASy Server. In The Proteomics Protocols Handbook; Walker, J.M., Ed.; Humana Press: Totowa, NJ, USA, 2005 ; pp. 571-607.

31. Smejkal, B.; Agrawal, N.J.; Helk, B.; Schulz, H.; Giffard, M.; Mechelke, M.; Ortner, F.; Heckmeier, P.; Trout, B.L.; Hekmat, D. Fast and scalable purification of a therapeutic full-length antibody based on process crystallization. Biotechnol. Bioeng. 2013, 110, 2452-2461. [CrossRef]

32. Hebel, D.; Ürdingen, M.; Hekmat, D.; Weuster-Botz, D. Development and Scale up of High-Yield Crystallization Processes of Lysozyme and Lipase Using Additives. Cryst. Growth Des. 2013, 13, 2499-2506. [CrossRef]

33. Teng, C.C.; Man, H.T. Simple reflection technique for measuring the electro-optic coefficient of poled polymers. Appl. Phys. Lett. 1990, 56, 1734-1736. [CrossRef]

34. Kabsch, W. XDS. Acta Crystallogr. Sect. D 2010, 66, 125-132. [CrossRef] [PubMed]

35. Winn, M.D.; Ballard, C.C.; Cowtan, K.D.; Dodson, E.J.; Emsley, P.; Evans, P.R.; Keegan, R.M.; Krissinel, E.B.; Leslie, A.G.; McCoy, A.; et al. Overview of the CCP4 suite and current developments. Acta Crystallogr. Sect. D Biol. Crystallogr. 2011, 67, $235-242$. [CrossRef] [PubMed]

36. McCoy, A.J.; Grosse-Kunstleve, R.W.; Adams, P.D.; Winn, M.D.; Storoni, L.C.; Read, R.J. Phaser crystallographic software. J. Appl. Crystallogr. 2007, 40, 658-674. [CrossRef] [PubMed]

37. Emsley, P.; Lohkamp, B.; Scott, W.G.; Cowtan, K. Features and development of Coot. Acta Crystallogr. Sect. D 2010, 66, 486-501. [CrossRef]

38. Murshudov, G.N.; Vagin, A.A.; Dodson, E.J. Refinement of Macromolecular Structures by the Maximum-Likelihood Method. Acta Crystallogr. Sect. D 1997, 53, 240-255. [CrossRef]

39. Schmidt, S.; Havekost, D.; Kaiser, K.; Kauling, J.; Henzler, H.-J. Crystallization for the Downstream Processing of Proteins. Eng. Life Sci. 2005, 5, 273-276. [CrossRef] 